\title{
Axion dark matter searches
}

\section{Yannis K. Semertzidis ${ }^{* 1,2}$}

1. Center for Axion and Precision Physics Research, IBS, Daejeon 34051, Republic of Korea

2. Department of Physics, KAIST, Daejeon 34141, Republic of Korea

E-mail: yannisaibs.re.kr, yannis@kaist.ac.kr

The axion field has recently undergone a qualitative transition with the establishment of major Centers around the world dedicated to its discovery. There are several critical improvements that made the entire axion mass range possible to probe with high sensitivity. It seems, with high probability, that within the next ten years we will know whether the axions are a significant part of the local dark matter for a large fraction of the allowed axion mass range.

The 39th International Conference on High Energy Physics (ICHEP2018)

4-11 July, 2018

Seoul, Korea

${ }^{*}$ Speaker. 
Two of the most important problems in present day standard model (SM): the so-called strong CP-problem, i.e., why the experimental limit on the neutron EDM is more than nine orders of magnitude smaller than naively expected from quantum-chromo-dynamics (QCD), and the question of what is the dark matter, might have a common solution in the axion field. Peccei and Quinn suggested [1] that the troublesome term in QCD could be dynamically balanced by a mechanism exploiting allowable symmetries. Weinberg [2] and Wilczek [3] suggested that the price to pay for this mechanism is the existence of a pseudoscalar particle dubbed the axion, with a mass inversely proportional to the PQ-mechanism scale. Initially it was thought that this scale is around the EW-mass level, however, this possibility was experimentally ruled out soon afterwards. Jihn E. Kim [4] suggested a new model which allows the axion mass to become much lighter, with much smaller coupling constant and thus evading the experimental limits, which he called "the invisible axion". This axion could also be an ideal cold dark matter candidate providing the required mass density to keep the stars in the galaxy together even though they travel much faster than allowed by Newtonian/Einstein gravity. Soon afterwards more models are published [5, 6, 7] and two major models, the KSVZ and DFSZ from the initials of the authors, became the standard goals of the axion experimental sensitivities. For recent theoretical overviews see [8,9].

The axions mix with $\pi^{0}$ and decay to two photons with a lifetime of about $10^{50}$ seconds for $10 \mu \mathrm{eV}$ axion mass. It couples to electromagnetism, to gluon fields, and the gradient of the axion field behaves as a, so-called, pseudo-magnetic field by its coupling to spins, see Figure (1).

\section{Axion Couplings}

- Gauge fields:

- Electromagnetic fields $L_{\text {int }}=-\frac{g_{a v}}{4} a F^{\mu \nu} \tilde{F}_{\mu \nu}=g_{a \gamma} a \vec{E} \cdot \vec{B}$

- Gluon Fields (Oscillating EDM,...)

$$
L_{\mathrm{int}}=\frac{a}{f_{a}} G_{\mu \nu} \tilde{G}^{\mu v}
$$

- Fermions (coupling with axion field gradient, pseudomagnetic field)

$$
L_{\mathrm{int}}=\frac{\partial_{\mu} a}{f_{a}} \bar{\Psi}_{f} \gamma^{\mu} \gamma_{5} \Psi_{f}
$$

Figure 1: There are several ways the axions couple with ordinary matter: 1. Electromagnetic, where in the presence of a strong magnetic field the axion field mixes with an electric field oscillating at the same frequency, 2. Coupling to gluon fields, creating oscillating electric dipole moments, and 3. The gradient of the axion field behaving like a pseudo-magnetic field. The axion field coherence length (De Broglie wavelength) is approximately $1 \mathrm{~m}$ for an axion mass of $1 \mathrm{meV}$ and it is inversely proportional to the axion mass. 


\section{Past efforts}

The main experimental method looking for axion conversion in a magnetic field is based on Sikivie's haloscope method $[10,11]$, i.e. the inverse Primakoff effect inside a resonator immersed in a large magnetic field. The same physics effect can be described as axion-electric field mixing in the presence of a strong magnetic field with same result [12]. The generated E-field, depending on the boundary and resonance conditions, can be enhanced by the quality factor of the resonator. The power of the axion to photon conversion is given by:

$$
P_{a \rightarrow \gamma}=\left(\frac{g_{\gamma} \alpha}{\pi f_{a}}\right)^{2} V B_{0}^{2} \frac{\rho_{a}}{m_{a}} C \min \left(Q_{L}, Q_{a}\right)
$$

where $Q_{a} \simeq 3 \times 10^{6}, V$ is the cavity volume, $Q_{L}$ is the loaded cavity quality factor for the $\mathrm{TM}_{010}$ mode, $\rho_{a}$ is the galactic halo axion density on Earth, and $m_{a}$ is the axion mass. The term $C$ is a geometrical form factor that depends on the mode:

$$
C=\frac{\left|\int_{V} d^{3} \vec{r} \vec{E}_{\omega} \cdot \vec{B}_{0}\right|^{2}}{B_{0}^{2} V \int_{V} d^{3} \vec{r} \varepsilon_{r}(\vec{r})\left|\vec{E}_{\omega}\right|^{2}},
$$

where $\varepsilon_{r}(\vec{r})$ is the dielectric constant in the cavity. For the $\mathrm{TM}_{010}$, the form factor can be evaluated as $C=0.69$, with smaller values for higher $\mathrm{TM}_{0 m 0}$ modes. The total axion to photon conversion power is given by

$$
\begin{aligned}
P & =2 \times 10^{-24} \text { Watt } \\
& \times\left(\frac{V}{5 \text { litter }}\right)\left(\frac{B_{0}}{7 \text { Tesla }}\right)^{2}\left(\frac{C}{0.4}\right)\left(\frac{g_{\gamma}}{0.36}\right)^{2} \frac{\rho_{a}}{5 \times 10^{-25} \mathrm{gr} / \mathrm{cm}^{3}}\left(\frac{m_{a} c^{2}}{v_{a} \mathrm{GHz}}\right)\left(\frac{Q_{L}}{10^{5}}\right),
\end{aligned}
$$

The first major attempt to discover the axions as part of the dark matter was an experiment at Brookhaven National Laboratory in 1987-92 by the Rochester, Brookhaven Fermilab (RBF) collaboration [13]. A conventional 6-7.5 T superconducting magnet of up to $10 \mathrm{~cm}$ aperture was used together with an RF-amplifier with a 8-15 K noise temperature, all running at liquid helium $4 \mathrm{~K}$, resulting to a total noise of about $15 \mathrm{~K}$ [14]. The sensitivity was 1 to 2 orders of magnitude higher than required to reach the DFSZ level. Soon afterwards, the University of Florida also published a more narrow line capitalizing in integrating out the noise by long averages in time, using essentially a similar experimental method [15]. Given that these efforts were the first attempts into searching for axion dark matter, it was amazing that they could reach so close to the theoretically expected levels. It was also very clear that to make the required improvement to reach all the way down to DFSZ, there would have to be major technological improvements.

The next collaboration for an axion dark matter experiment (ADMX) was formed around 1992, basing their goals into getting better SQUID-based RF-amplifiers, developed by John Clarke with useful frequencies up to $1 \mathrm{GHz}$ [16]. An additional breakthrough was the fact that dilution refrigerators became much more efficient and easier to operate, so temperatures below $0.1 \mathrm{~K}$ became possible. The prospects of a low frequency $(<1 \mathrm{GHz})$ low-noise amplifier also set the radius of the resonant cavity and thus its volume. The ADMX schematic, with the outlining of the new improvements brought in, is shown in Figure (2), and the sensitivities of all those experiments are shown in Figure (3). 


\section{Axion Dark Matter e.Xperiment}

- Cryogenics (0.1K)

- Superconducting magnet $(>7.5 \mathrm{~T})$

- Large volume cavity (140l)

- Low noise amplifiers

- From $12 \mathrm{~K}$ to $1 \mathrm{~K}$

- Currently SQUID and JPA $(<1 \mathrm{~K})$

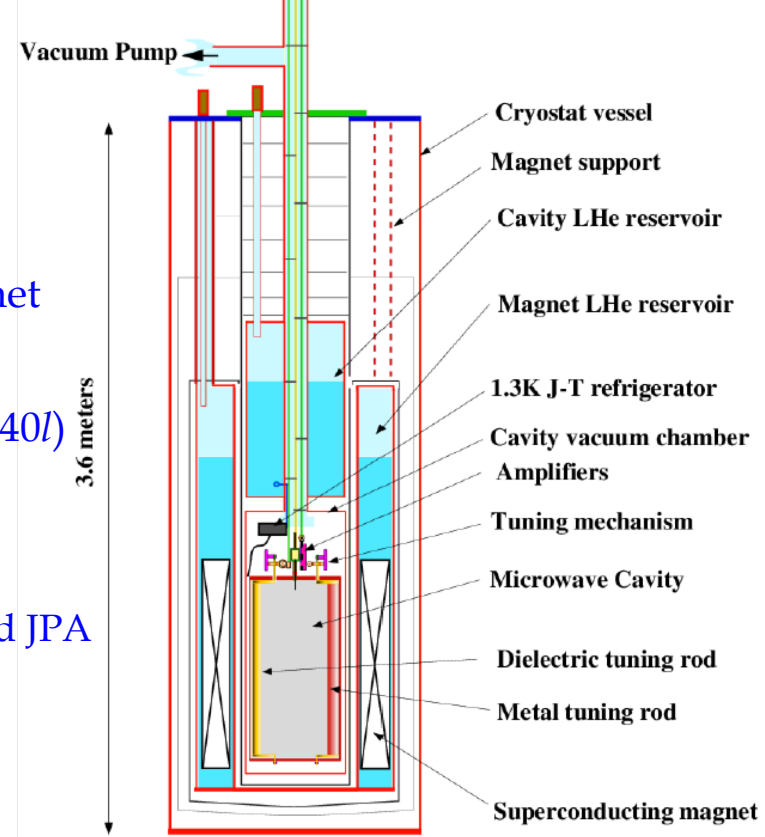

Figure 2: The ADMX schematic and its improvements that made it possible to have better sensitivity than the previous efforts.

\section{Current efforts}

Since we do not know the axion mass, we need to scan all possible axion frequencies. Using Dicke's radiometer equation [17]

$$
S N R=\frac{P_{\text {sig }}}{k_{B} T_{s y s}} \sqrt{\frac{t}{\Delta v_{a}}}
$$

we can estimate the axion frequency scanning rate

$$
\begin{aligned}
\frac{d f}{d t}=\frac{f}{Q_{L}} \frac{1}{t} & \approx \frac{1 \mathrm{GHz}}{\text { year }}\left(g_{a \gamma \gamma} 10^{15} \mathrm{GeV}\right)^{4} \\
& \times\left(\frac{5 \mathrm{GHz}}{v_{a}}\right)^{2}\left(\frac{4}{S N R}\right)^{2}\left(\frac{0.25 \mathrm{~K}}{T}\right)^{2}\left(\frac{B}{25 \mathrm{~T}}\right)^{4}\left(\frac{C}{0.6}\right)^{2}\left(\frac{V}{5 l}\right)^{2}\left(\frac{Q_{L}}{10^{5}}\right)
\end{aligned}
$$

with $T=T_{\text {electronic }}+T_{\text {physical }}$, i.e., the sum of the electronics plus physical temperatures of the system. When it is done properly, it is dominated by the electronic noise of the first amplifier and the physical temperature of the cavity. The physical temperature could go down to $50 \mathrm{mK}$ even for a large system, the magnetic field could go up to $25 \mathrm{~T}$ using high temperature superconducting (HTS) magnet technology, and even further up to 35-40 $\mathrm{T}$ when combined with low temperature superconducting (LTS) magnets. Other improvements can result by phase-matching several axion dark matter experiments even offline, and finally by improving the quality factor of the microwave resonator, e.g, using superconducting thin film or superconducting tapes of HTS. Clearly, the technology is here and, when all these improvements are combined, can increase the sensitivity to tell whether or not axions are locally $100 \%$ of the estimated average dark matter density. 


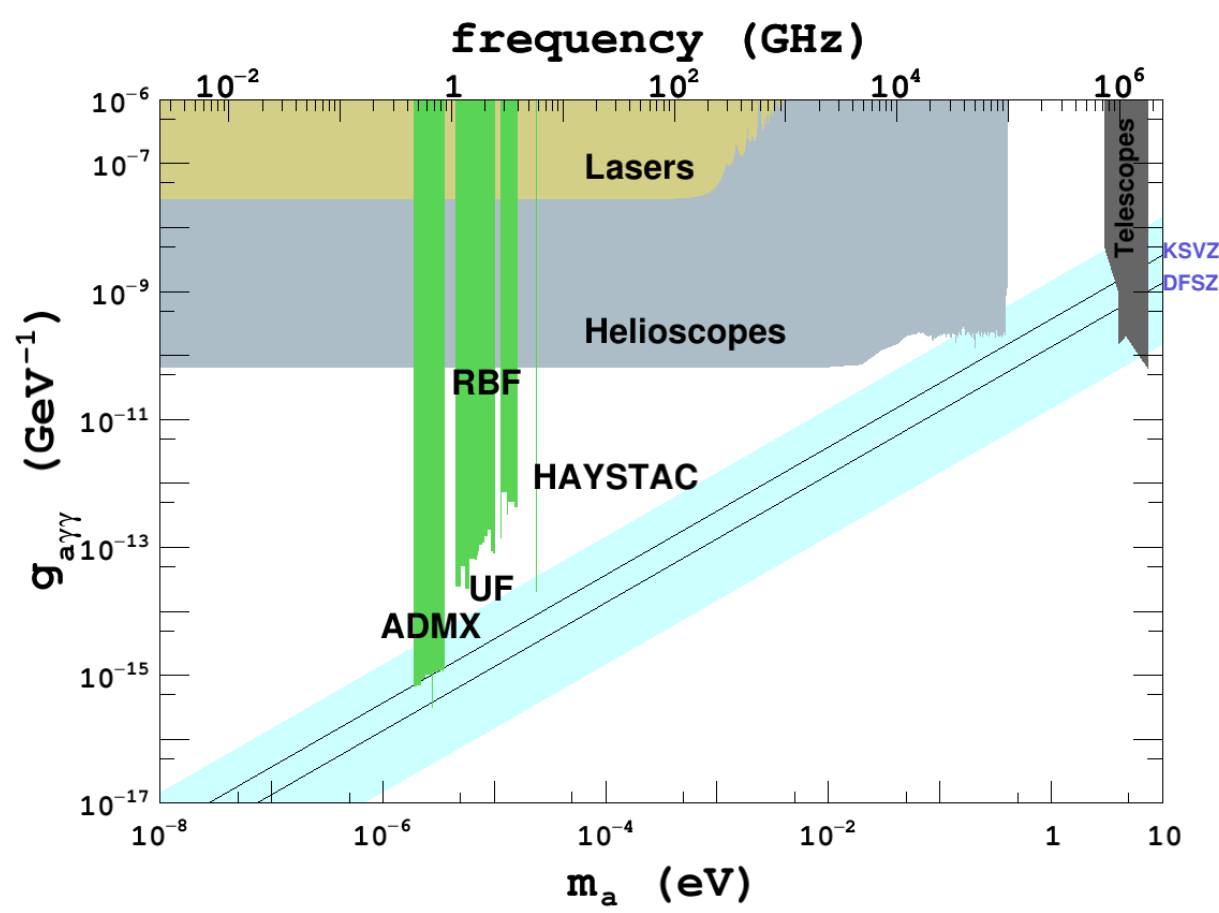

Figure 3: The axion coupling to two photons vs. the axion mass is shown here as well as the limits from various experimental methods. The KSVZ and DFSZ lines represent two models, which solve the Strong$\mathrm{CP}$ problem and the colored band denotes the overall uncertainty. Axion like particles (ALPs) are named possible particles, which may be discovered to have a coupling constant and a mass outside the band defined by the KSVZ and DFSZ lines, and thus they don't solve the Strong-CP problem.

The possible axion mass range is too large to be able to cover it entirely using a single technology. Several ideas have been developed lately giving hope that it will be possible to achieve the required sensitivities for the case where dark matter is composed entirely of axions. Some of the efforts based on the $a\left(\omega_{0}\right) \vec{E} \cdot \vec{B}$ coupling and promising to reach all the way to DFSZ level are:

- ADMX, with a frequency range of $0.4-10 \mathrm{GHz}$, with the lowest frequency having the highest priority and most likely to happen first.

- IBS/CAPP, a.k.a. CULTASK (CAPP's Ultra-Low Temperature Axion Search in Korea), with a possible frequency range of $0.7-10 \mathrm{GHz}$ in the first phase and $10-20 \mathrm{GHz}$ in the second phase. The $20-50 \mathrm{GHz}$ range may be possible by using open resonators.

- MADMAX, with a possible frequency range of $10-100 \mathrm{GHz}$ using high quality dielectrics and a large volume $10 \mathrm{~T}$ magnet.

- ABRACADABRA [18], and DM-Radio [19], which can be sensitive to low frequency axions by looking for AC-magnetic flux induced by a DC-magnetic field interacting with the axion 
dark matter field.

while some of the experiments based on different couplings are

- ARIADNE, which is sensitive to monopole-dipole interactions without requiring the existence of axion dark matter, only of the axion physics,

- GNOME, a global network of optical magnetometers, sensitive to axion field gradients,

- CASPEr, an oscillating EDM experiment using a DC magnetic field while the axions oscillate at the Larmor frequency of nuclei and the Axion-Storage-ring-EDM, which can run parasitically to the storage-ring proton EDM experiment.

Of the above projects, ARIADNE is not haloscope based experiment, i.e., it does not require the existence of axion dark matter. However, for ARIADNE [20] to have a chance to observe a signal, there must be at least an additional CP-violating phase, e.g., due to SUSY, which will cause enough offset into the $\theta_{Q C D}$ to be within its experimental sensitivity. The potential phase space available to ARIADNE is shown in Figure (4). The upper limit of the available phase-space is defined by the current experimental limit on the neutron EDM (nEDM) of $3 \times 10^{-26} e \cdot \mathrm{cm}$, while the lower one is restricted by the electro-weak (EW) CP-violating phase, corresponding to about $10^{-31} e \cdot \mathrm{cm}$ for the pEDM and the nEDM. If, e.g., the proton and/or neutron EDM is observed to be above $10^{-27} e \cdot \mathrm{cm}$, and the ARIADNE experiment does not observe a signal with the currently projected sensitivity, it would mean the exclusion of the axion in a large mass range, as shown in Figure (4). This is particularly important for the high mass range, $0.1-10 \mathrm{meV}$, which is particularly difficult to probe as dark matter.

Other ongoing experiments or under preparation improving their performance are

- Light Shining Through the Wall experiments, attempt to observe axions by producing them in a dipole magnetic field using intense laser light and then stopping the laser light by a thick opaque wall, while axions travel through. A symmetric system is then used to back convert the axions into observable photons. Since there is a requirement to first produce and then convert them back, the probability goes as $g_{a \gamma \gamma}^{4}$ and hard to make progress fast. The conversion probability also goes as $B^{2} L^{2}$, the magnetic field strength and its length correspondingly. A recent conceptual improvement calls for phase locking the produced axions in the first dipole and the converted photons in the second dipole greatly improving its prospects [21].

- Helioscopes, with CAST the last helioscope [22], running at CERN, with new proposals for major investments and improvements named IAXO [23] and BabyIAXO as a first step. The helioscopes are based on the fact that the center of the sun can produce axions very efficiently. The produced axion field is a plane wave which can be converted back to x-rays inside a dipole magnetic field, see Figure (5), here on earth. 


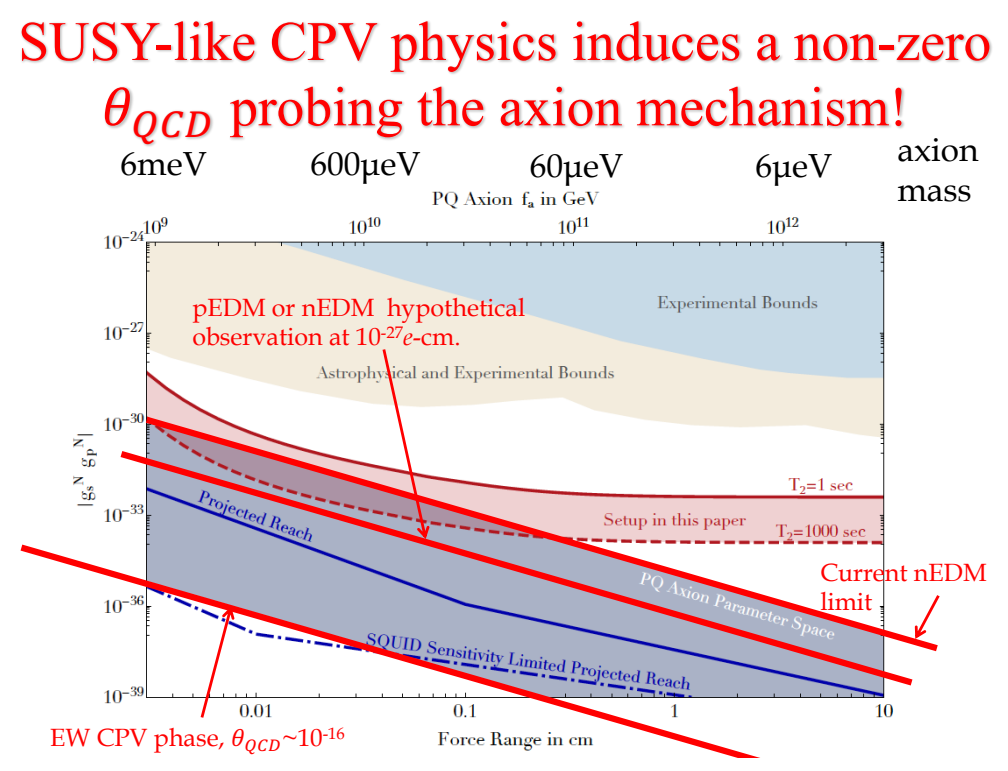

Figure 4: The CP-violating couplings vs. the axion mass that will be available to be probed by the ARIADNE experiment. The upper limit corresponds to the current neutron EDM (nEDM) experimental limit, while the lower limit corresponds to the EW, CP-violating phase. In case the proton and/or neutron EDM is observed at the $10^{-27} e \cdot \mathrm{cm}$ level or above and ARIADNE does not observe any signal at its currently projected sensitivity, then this would rule-out a large range of possible axion masses. The range of 0.1 $10 \mathrm{meV}$ may be too difficult to probe directly otherwise.

\subsection{ADMX}

The recent achievement of ADMX reaching all the way to the DFSZ sensitivity is a major accomplishment [24]. The frequency reach of the present system is up to $2 \mathrm{GHz}$ with high sensitivity. The ADMX collaboration decided to expand the axion frequency by phase matching individual cavities within the large magnet volume based on JPAs and large $B^{2} V$. The challenges of the phase-matching of several cavities together have been overcome by recent work and there is every reason to believe that this work, even though challenging, will proceed as planned. Work on open resonators promise to expand the axion frequency sensitivity up to $50 \mathrm{GHz}$ [25].

\subsection{IBS/CAPP}

The new Center in Korea [26] is basing its research on commissioning the largest magnetic fields possible for relatively large apertures: a $25 \mathrm{~T}$, HTS magnet with $100 \mathrm{~mm}$ aperture (HTS$25 \mathrm{~T} / 100 \mathrm{~mm}$ ) and a $12 \mathrm{~T}$, LTS, based on $\mathrm{Nb}_{3} \mathrm{Sn}$ cable, with $320 \mathrm{~mm}$ aperture (LTS-12T/320 mm). An overview of the state of the art magnets available for the axion dark matter experiments and of their figure of merit in various applications is given in [27]. Additional improvements can be realized by achieving the highest possible quality factor copper-cavities as well as doing R\&D on developing superconducting cavities that can withstand a penetration of high magnetic fields without suffering loss in RF-quality factor. Using the lowest physical temperature for the microwave resonator and developing the best RF-amplifiers possible, using currently available technology, will allow to achieve sensitivity all the way down to DFSZ scale for a significant fraction of axions to 


\section{Solar Axions}

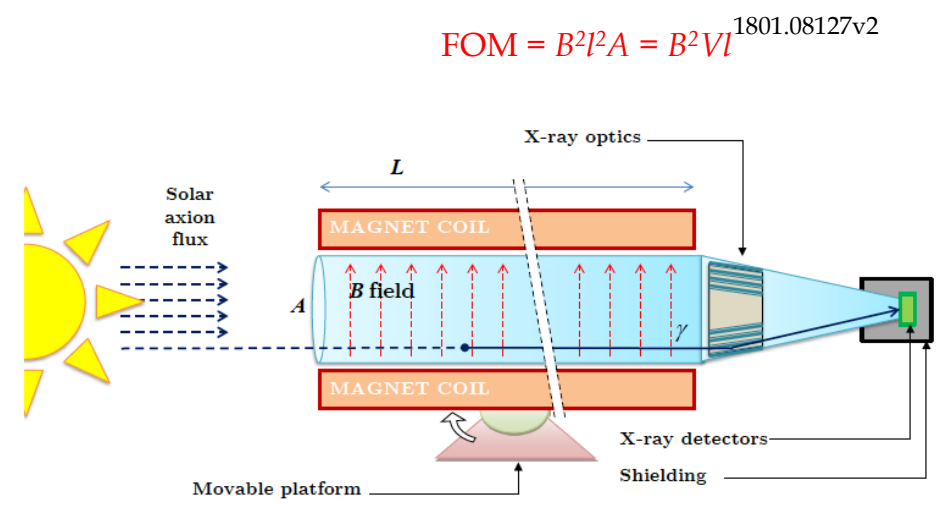

Figure 5: Solar axions travel to earth in a form of a plane wave, which mixes with photons in the presence of a magnetic field. The axion to photon conversion spectrum is in the $2-5 \mathrm{keV} x$-rays, reflecting the core temperature of the sun.

the dark matter content and an axion frequency range of $0.7-10 \mathrm{GHz}$ using a single cavity, shown in Figure (6). In the next phase the experiment will use newly developed techniques at IBS/CAPP to achieve high efficiency, high frequency axion sensitivity, up to $20 \mathrm{GHz}$. The development of a single photon above about $5 \mathrm{GHz}$ will further increase the sensitivity to axion dark matter significantly.

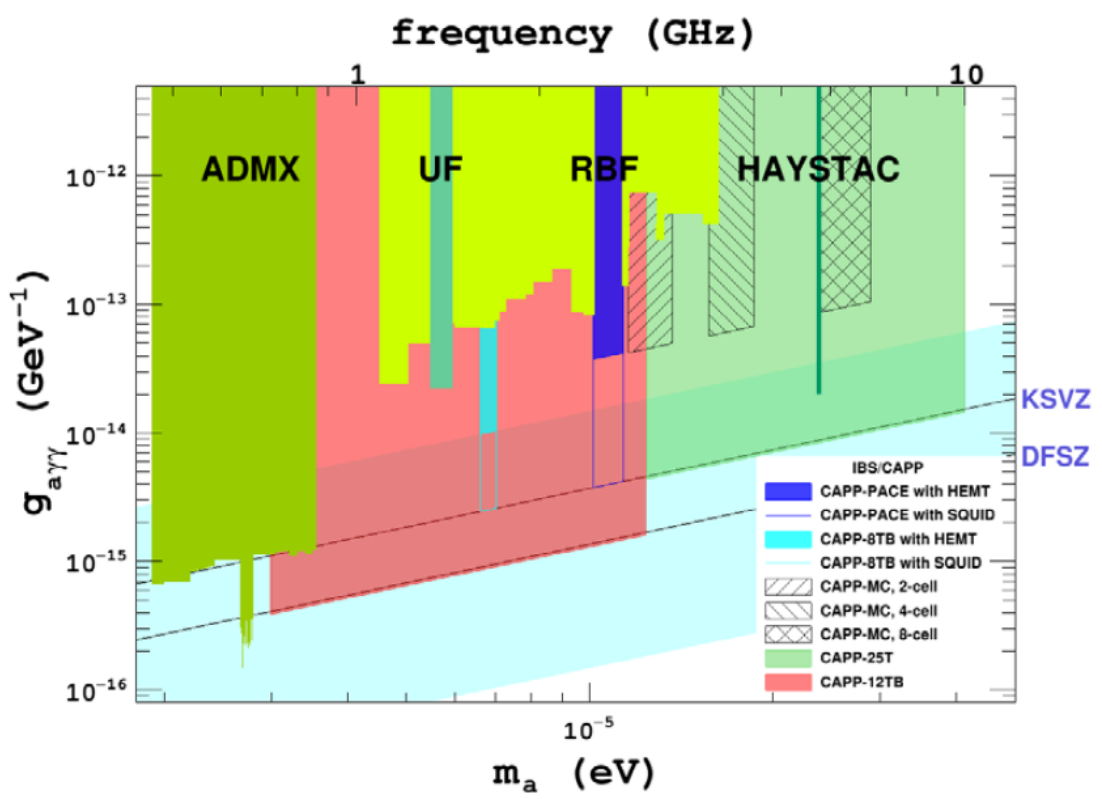

Figure 6: CAPP's CULTASK projected sensitivity based on currently running axion dark matter experiments as well as the future ones based on single cavities and the HTS, $25 \mathrm{~T}$ with $100 \mathrm{~mm}$ aperture magnet and the LTS, $\mathrm{Nb}_{3} \mathrm{Sn}$ based, $12 \mathrm{~T}$ with $320 \mathrm{~mm}$ aperture magnet. 


\subsection{MADMAX}

A fundamental problem of the cylindrical microwave cavities is that at frequencies above $20 \mathrm{GHz}$ they become impractical, due to very small radius. A new method, based on the axionphoton mixing in the presence of a magnetic field and the reflection of the photon at a medium with high dielectric constant, provides an alternative way of searching for axion dark matter in the high frequency range, shown in Figure (7). This method is applied to take full advantage of the large $B^{2} V$ of large volume $[28,29,30]$ magnetic fields and yet be sensitive to high frequency axions with a range of $10-100 \mathrm{GHz}$.

\section{B. Majorovits}

\section{Experimental approaches: Effect of Dielectric}

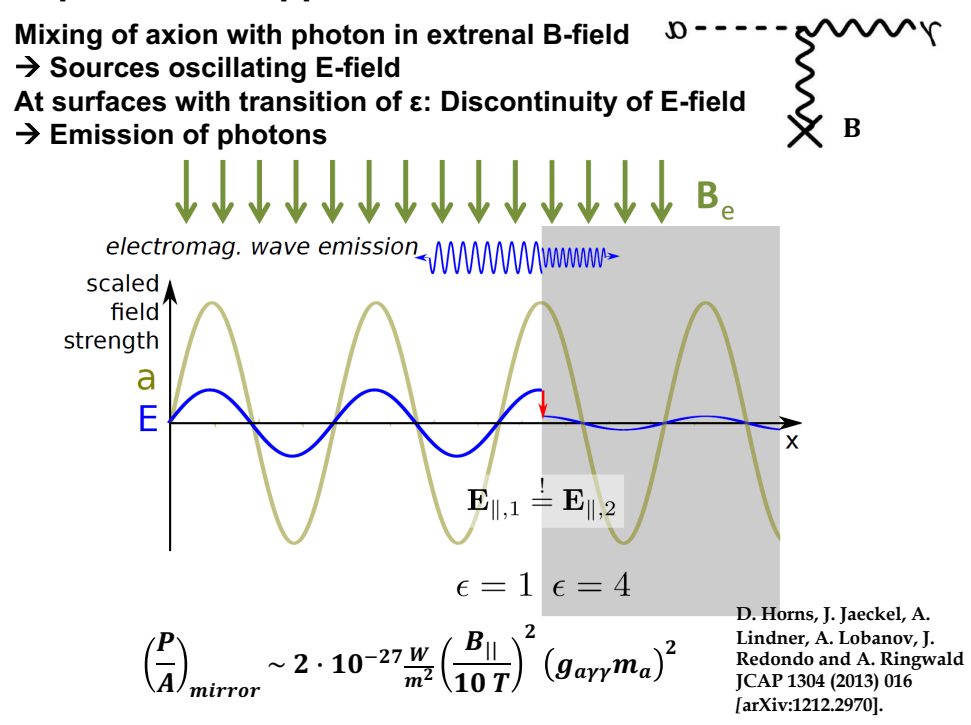

Figure 7: The axions mix with photons in the presence of a magnetic field. The electric field is reflected at the interface of vacuum/medium due to the large dielectric constant of the medium. The method is a brilliant demonstration of an efficient application of the $B^{2} V$ parameters for a large axion mass.

\subsection{Oscillating EDMs}

Even though the vacuum $\theta_{\mathrm{QCD}}$ average value is very small, nonetheless, its oscillation has an effect on the EDM of the proton and neutron and other nuclei in general [31, 32]. An experiment, named CASPEr [33], based on the NMR resonance with the EDM oscillation frequency can eventually reach all the way down to theoretically interesting sensitivity levels, see Figure (8). Another idea, based on the resonance between the axion dark matter oscillation frequency and the proton or deuteron $g-2$ frequency, using the storage ring EDM method, is also possible [34].

\subsection{Summary}

The field of axion dark matter is developing at a healthy rate and there are several projects that promise to reach all the way to the theoretically interesting levels covering almost all the relevant axion mass range, see Figure (9), nicely summarized in a recent overview by Irastorza 


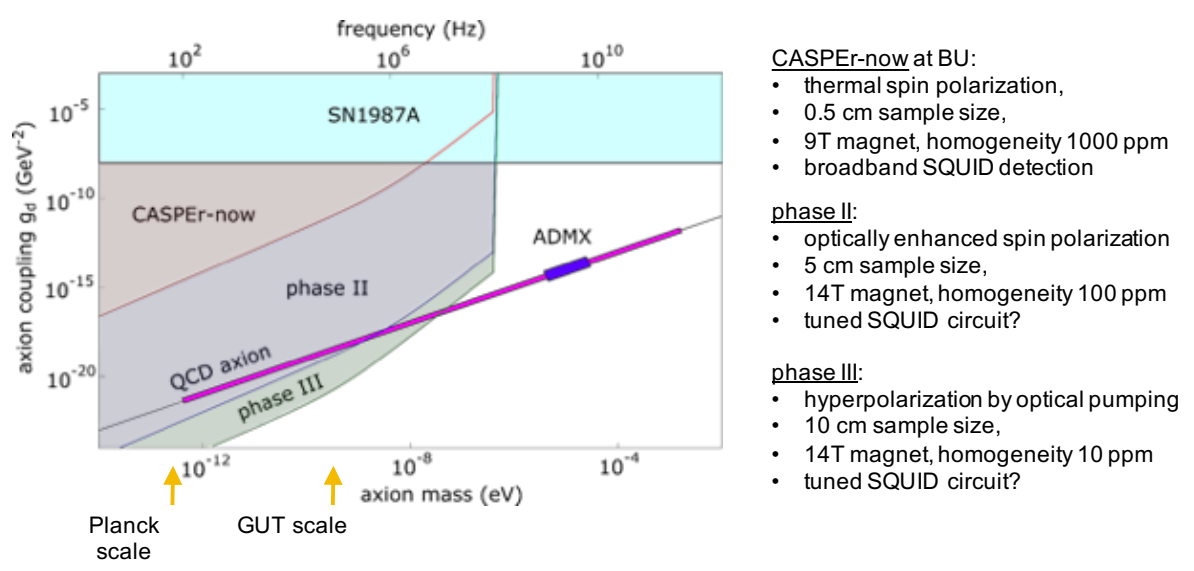

Figure 8: The axion mass originates from the oscillating $\theta_{\mathrm{QCD}}$ term and it corresponds to the same frequency. That same frequency also causes the oscillation of the EDM in nuclei, which can be probed by an NMR like method using an appropriate bulk material.

and Redondo [35]. In the next five to ten years, it is most likely that we will know whether or not axions contribute a significant part of the local dark matter, while their discovery could be announced at any moment. Their discover will also signify the start of a new era, that of axion astrophysics, providing us a window into the early galaxy formation dynamics and the current dark matter distribution.

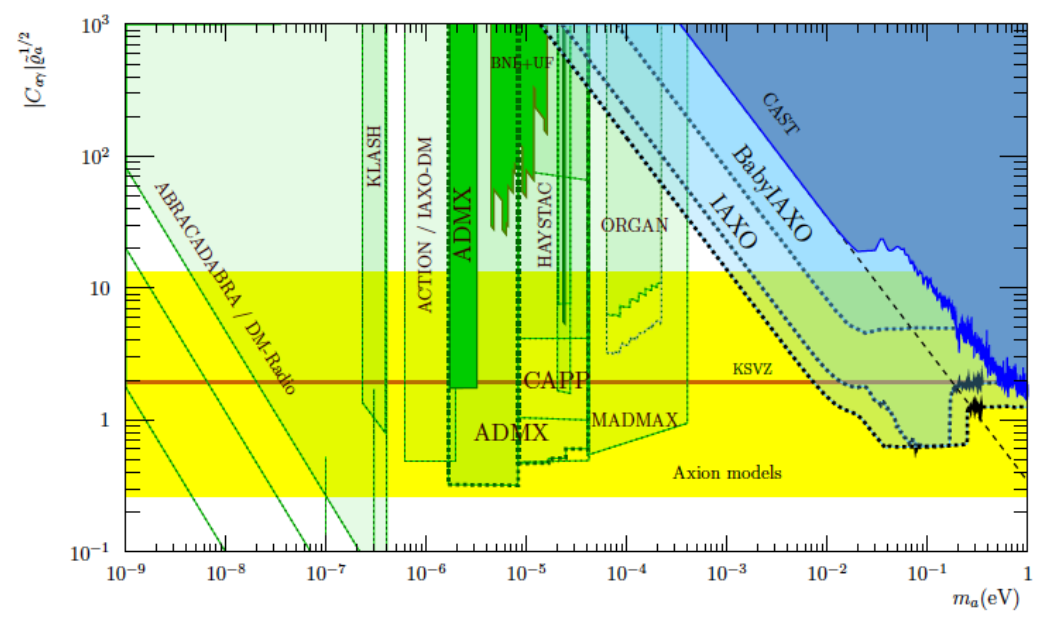

Figure 9: The axion coupling vs. mass projected sensitivities from various efforts either current or in the design phase, from reference [35].

\section{References}

[1] R. Peccei and H. Quinn, Phys. Rev. Lett. 38, 1440 (1977).

[2] S. Weinberg, Phys. Rev. Lett. 40, 223 (1978).

[3] F. Wilczek, Phys. Rev. Lett. 40, 279 (1978). 
[4] J. E. Kim, Physical Review Letters 43, 103 (1979).

[5] M. Shifman, A. Vainshtein, and V. Zakharov, Nuclear Physics B 166, 493 (1980).

[6] A. R. Zhitnitsky, Sov. J. Nucl. Phys. 31, 260 (1980).

[7] M. Dine, W. Fischler, and M. Srednicki, Physics Letters B 104, 199 (1981).

[8] P. W. Graham et al., Annual Review of Nuclear and Particle Science 65, 485 (2015).

[9] J. Kim, S. Nam, and Y. Semertzidis, International Journal of Modern Physics A, 33, 1830002 (2017).

[10] P. Sikivie, Phys. Rev. Lett. 51, 1415 (1983).

[11] P. Sikivie, Phys. Rev. D 32, 2988 (1985).

[12] Jooyoo Hong et al., "Calculations of resonance enhancement factor in axion-search tube-experiments," arXiv:1403.1576, 2014.

[13] W.U. Wuensch et al., Phys. Rev. D 40, 3153 (1989).

[14] S. De Panfilis and J. Rogers, IEEE Trans. Microwave Theory Tech. 36, 607 (1988).

[15] C. Hagmann et al., Phys.Rev. D42 (1990) 1297-1300.

[16] J. Clarke and A. I. Braginski, The SQUID handbook: Applications of SQUIDs and SQUID systems (John Wiley \& Sons, 2006).

[17] R. H. Dicke, Review of Scientific Instruments 17, 268 (1946).

[18] Y. Kahn, B. R. Safdi, and J. Thaler, Phys. Rev. Lett. 117 no. 14, (2016) 141801, arXiv:1602.01086

[19] M. Silva-Feaver et al., "Overview of the DM Radio Pathfinder Experiment," arXiv:1610.09344, 2016.

[20] A. Arvanitaki and A.A. Geraci, Phys. Rev. Lett. 113 (2014) no.16, 161801

[21] R. Bahre et al., “Any Light Particle Search II” Technical Design Report, arXiv:1302.5647, 2013.

[22] CAST Collaboration, V. Anastassopoulos et al., Nature Phys. 13 (2017) 584-890.

[23] E. Armengaud et al., "Design of the International Axion Observatory (IAXO)," JINST 9 (2014) T05002, arXiv:1401.3233

[24] ADMX Collaboration, N. Du et al., Phys. Rev. Lett. 120, 151301 (2018).

[25] G. Rybka et al., Phys. Rev. D91 no. 1, (2015) 011701, arXiv:1403.3121

[26] https : //capp.ibs.re.kr/html/capp_en/.

[27] R. Battesti et al., Phys. Rept. 765-766 (2018) 1-39.

[28] P. F. Smith, Proceedings, $7^{\text {th }}$ Moriond Workshop, Les Arcs, France, January 24-31, 1987, pp. 233-242, 1987.

[29] A. J. Millar et al., JCAP 1701 no. 01, (2017) 061, arXiv:1612.07057 [hep-ph].

[30] MADMAX Working Group Collaboration, A. Caldwell et al., Phys. Rev. Lett. 118 no. 9, (2017) 091801, arXiv:1611.05865.

[31] P. W. Graham and S. Rajendran, Phys. Rev. D 84, 055013 (2011).

[32] P. W. Graham and S. Rajendran, Phys. Rev. D 88, 035023 (2013).

[33] D. Budker et al., Phys.Rev. X4 (2014) 021030, arXiv:1306.6089

[34] S.P. Chang et al., "Axion dark matter search using the storage ring EDM method," arXiv:1710.05271, 2017.

[35] I. Irastorza and J. Redondo, Prog. Part. Nucl. Phys. 102 (2018) 89-159, arXiv:1801.08127v2 